Pacific Journal of Mathematics

BONDED QUADRATIC DIVISION ALGEBRAS 


\section{BONDED QUADRATIC DIVISION ALGEBRAS}

\section{R. A. CZerwinski}

Osborn has shown that any quadratic algebra over a field of characteristic not two can be decomposed into a copy of the field and a skew-commutative algebra with a bilinear form. For any nonassociative algebra $G$ over a field of characteristic not two, Albert and Oehmke have defined an algebra over the same vector space, which is bonded to $G$ by a linear transformation $T$. In this paper this process is specialized to the class $\mathscr{A}$ of finite dimensional quadratic algebras $A$ over fields of characteristic not two, which define a symmetric, nondegenerate bilinear form, to obtain quadratic algebras $B(A, T)$ bonded to $A$. In the main results $T$ will be defined as a linear transformation on the skew-commutative algebra $V$ defined by Osborn's decomposition of $A$. An algebra in $\mathscr{A}$ is called a division algebra if $A \neq 0$ and the equations $a x=b$ and $y a=b$, where $a \neq 0$ and $b$ are elements in $A$, have unique solutions for $x$ and $y$ in $A$. Consequently, a finite dimensional algebra $A \neq 0$ is a division algebra if and only if $A$ has no divisors of zero. A basis for $V$ is said to be orthogonal, if it is orthogonal with respect to the above mentioned bilinear form. An algebra in $\mathscr{A}$ is weakly flexible if the $i$ th component of the skew-commutative product of the $i$ th and $j$ th members of each orthogonal basis of $V$ is 0 . If $\mathscr{D}(\mathscr{A})$ denotes the class of division algebras in $\mathscr{A}$ and $I$ denotes the identity transformation on $V$, then the main results are: (1) $A \in \mathscr{D}(\mathscr{A}), \quad T$ nonsingular and $B(A, T)$ flexible imply $B(A, T) \in \mathscr{D}(\mathscr{A}),(2)$ if $A \in \mathscr{D}(\mathscr{A})$ and $A$ is weakly flexible, then $B(A, T)$ is weakly flexible if and only if $T=\delta I$ for $\delta$ a scalar, and (3) if $A$ is a Cayley-Dickson algebra in $\mathscr{D}(\mathscr{A})$, then $B(A, T)$ is a Cayley-Dickson algebra in $\mathscr{D}(\mathscr{A})$ if and only if $T= \pm I$. Finally, a class of nonflexible quadratic division algebras bonded to Cayley-Dickson division algebras will be exhibited.

1. Introduction. A finite dimensional algebra $A$ with identity element 1 over a field $F$ of characteristic not 2 is called a quadratic algebra in case $1, a$, and $a^{2}$ are linearly dependent over $F$ for all $a \in A$. Following the conventions used by Osborn [6] we shall identify the field $F$ with the subalgebra $F 1$ and refer to an element in $F 1$ as a scalar. Furthermore, if an element $x \in A$ squares to a scalar but $x$ is not a scalar, $x$ is called a vector. If $V$ is the set of all vectors in $A$, then $A$ is a vector space direct sum of $F$ and $V$. For $x$ and $y \in A$, let $(x, y)$ denote the scalar component of $x y$. Clearly $(x, y)$ is a bilinear form from $A \times A$ 
to $F$. If $x$ and $y \in V$, we define " $\times$ " by $x \times y=x y-(x, y), V$ is closed under this product and Osborn [6, p. 203] shows it is skewcommutative. If $\alpha+x$ and $\beta+y \in A=F+V$, where $\alpha$ and $\beta \in F$ and $x$ and $y \in V$, then

$$
(\alpha+x)(\beta+y)=[\alpha \beta+(x, y)]+[\alpha y+\beta x+x \times y] \in F+V .
$$

This decomposition of $A$ into a copy of the field and a skew-commutative algebra with bilinear form makes it possible to restate questions about quadratic algebras in terms of questions about bilinear forms and skew-commutative algebras. For example, it is easy to show that $A$ satisfies the flexible law if and only if the bilinear form $(x, y)$ is symmetric and $(x, x \times y)=0$ for all $x$ and $y$ in $V$, and that $A$ is alternative if and only if $A$ is flexible and $(y, x) x-(x, x) y+(y \times x) \times x=0$ for all $x$ and $y$ in $V$.

Let $\mathscr{A}$ denote the class of algebras satisfying: $A$ is a finite dimensional quadratic algebra over a field $F$ of characteristic not two and $A$ defines a symmetric, nondegenerate bilinear form $(x, y)$. We call an algebra in $\mathscr{A}$ a division algebra if $A \neq 0$ and the equations $a x=b$ and $y a=b$, where $a \neq 0$ and $b$ are elements in $A$, have unique solutions for $x$ and $y$ in $A$. Consequently, a finite dimensional algebra $A \neq 0$ is a division algebra if and only if $A$ has no divisors of zero. Let $\mathscr{D}(\mathscr{A})$ denote the class of division algebras in $\mathscr{A}$. In the case that $(x, y)$ is defined by a division algebra it will be nondegenerate, since otherwise there exists an element $\alpha+x \in A$ such that $(y, \alpha+x)=0$ for all $y \in A$. But then $0=(x, \alpha+x)=(x, \alpha)+(x, x)=x^{2}$, which contradicts the division property of $A$.

The assumptions of finite dimensionality of $A$ and symmetry of $(x, y)$ are sufficient to prove $V$ has a basis $u_{1}, u_{2}, \cdots, u_{n}$ of mutually orthogonal vectors with respect to $(x, y)$. Henceforth, when we speak of an orthogonal basis for $V$, we shall always mean orthogonal with respect to the bilinear form $(x, y)$. Moreover, we will let $u_{i}^{2}=\alpha_{i} \in F$ for $i=1, \cdots, n$; and for $i \neq j$, let $u_{i} u_{j}=\sum_{k=1}^{n} \xi_{i j k} u_{k}$, so that the $\xi_{i j k}$ 's are the multiplication constants of an orthogonal basis of $V$ : Note that

$$
\sum_{k=1}^{n} \xi_{i j k} u_{k}=u_{i} u_{j}=u_{i} \times u_{j}=-u_{j} \times u_{i}=-u_{j} u_{i}=-\sum_{k=1}^{n} \xi_{j i k} u_{k}
$$

for all $i, j, k=1, \cdots, n$ and $i \neq j$. So

$$
\xi_{i j k}=-\xi_{j i k} \quad \text { for all nonzero } i, j \text {, and } k \text {. }
$$

If $(x, y)$ is nondegenerate, then $\alpha_{i} \neq 0$ for $i=1, \cdots, n$, since $\alpha_{i}=0$ implies $0=\left(u_{i}, u_{i}\right)$ which would imply $\left(u_{i}, y\right)=0$ for all $y \in A$. 
For $A \in \mathscr{A}$ let $U$ be the subspace of $A$ consisting of all finite linear combinations of vectors of the form $x y-y x$ for $x$ and $y \in A$. Let $T$ be a linear mapping from the subspace $U$ into $A$ and let $B(A, T)$ be an algebra with the same vector space as $A$ and multiplication defined by

$$
x \cdot y=\frac{1}{2}(x y+y x)+\frac{1}{2}(x y-y x)^{T},
$$

where $x y$ denotes multiplication in $A$. $T$ will be called a bonding mapping ([2] and [5]) of $A$ and $B(A, T)$ will be said to be bonded to $A$. Using (1.2) it is seen that powers in $B(A, T)$ agree with those in $A$ and that the identity of $A$ is also the identity in $B(A, T)$. Thus $B(A, T)$ is also a quadratic algebra and we will let $(x, y)_{T}$ denote the bilinear form defined as the scalar component of $x \cdot y$ in $B(A, T)$ and let $x \times_{T} y=$ $x \cdot y-(x, y)_{T}$, for all $x$ and $y \in V . \quad V$ is closed under this skewcommutative product. Since $(x, y)$ is assumed to be symmetric and $x \times y$ is skew-commutative, we have for all $x$ and $y \in V$ :

$$
\begin{aligned}
& \frac{1}{2}(x y+y x)=(x, y) \quad \text { and } \\
& \frac{1}{2}(x y-y x)=x \times y .
\end{aligned}
$$

So for all $x$ and $y \in V$ :

$$
x \cdot y=(x, y)_{T}+x \times_{T} y=(x, y)+(x \times y)^{T} .
$$

Clearly, for any basis $u_{1}, \cdots, u_{n}$ of $V$, the set of vectors $\left\{u_{i} \times u_{j} \mid i, j=\right.$ $1, \cdots, n\}$ spans the space $U \subseteq V$. Since most of our knowledge is obtained under the assumption that $T$ is a mapping into $V$, we will henceforth make the restriction

$$
\left(u_{i} \times u_{j}\right)^{T}=\sum_{k=1}^{n} \beta_{i j k} u_{k}
$$

The $\beta_{i j k}$ 's for $i, j, k,=1, \cdots, n$ are then the corresponding multiplication constants for $V$ in $B(A, T)$ and $(x, y)_{T}=(x, y)$.

2. Lemma 2.1. Let $A \in \mathscr{A}$ and let $u_{1}, \cdots, u_{n}$ be any orthogonal basis of $V$. Then $A$ is flexible if and only if

(a) $\xi_{i j i}=0$ for all $i, j=1, \cdots, n$ and

(b) $\xi_{i j k} \alpha_{k}=\xi_{k i j} \alpha_{j}=\xi_{j k i} \alpha_{i}$ for all $i, j, k$ distinct in $\{1, \cdots, n\}$.

Proof. By assumption $(x, y)$ is symmetric, so it suffices to show that the condition $0=(x, x \times y)$ for all $x$ and $y$ in $V$ is equivalent to conditions (a) and (b). The condition $0=(x, x \times y)$ is equivalent to the 
linearization $0=(x, z \times y)-(z, x \times y)$, and by the linearity of this relation it is equivalent to the set of equations

$$
\begin{aligned}
0 & =\left(u_{i}, u_{j} \times u_{k}\right)+\left(u_{j}, u_{i} \times u_{k}\right) \\
& =\left(u_{i}, \xi_{j k i} u_{i}\right)+\left(u_{j}, \xi_{i k j} u_{j}\right) \\
& =\xi_{j k i} \alpha_{i}+\xi_{i k j} \alpha_{j},
\end{aligned}
$$

for all $i, j, k \in\{1, \cdots, n\}$. The latter conditions are condition (a) of the theorem when $k=i$ or $j$, and condition (b) when $i, j$, and $k$ are distinct.

We shall call $A \in \mathscr{A}$ weakly flexible if property (a) in Lemma 2.1 is satisfied for each orthogonal basis of $V$. Osborn [6, pp. 204-206] calls a skew-commutative algebra $V$ division-like if there do not exist linearly independent $u$ and $v \in V$ such that $u \times v=0$ or $u \times v=u$ and he shows that $A=F+V$ is a division algebra if and only if $V$ is division-like and a certain condition is satisfied by its bilinear form.

LEMma 2.2. Let $A \in \mathscr{D}(\mathscr{A})$. A is weakly flexible if and only if for $x$ and $y \in V$ such that $(x, y)=0$, there exists $z \in V$ such that $x=y \times z$.

Proof. Suppose first that $A$ is weakly flexible. Since $(x, y)=0$, there exists an orthogonal basis $u_{1}=x, u_{2}=y, u_{3}, \cdots, u_{n}$ for $V$. Since $A \in \mathscr{D}(\mathscr{A})$, there exists $\alpha+z \in A$ such that

$$
u_{1}=u_{2}(\alpha+z)=\alpha u_{2}+\left(u_{2}, z\right)+u_{2} \times z=\alpha u_{2}+u_{2} \times z .
$$

Let $z=\sum_{j=1}^{n} \gamma_{j} u_{j}$. Then

$$
u_{2} \times z=\sum_{j=1}^{n} \gamma_{j}\left(u_{2} \times u_{j}\right)=\sum_{k=1}^{n} \sum_{j=1}^{n} \gamma_{j} \xi_{2 j k} u_{k}
$$

Since $A$ is weakly flexible, $\xi_{2 j 2}=0$ for all $j=1, \cdots, n$, so the coefficient of $u_{2}$ in $u_{2} \times z$ is 0 , which then implies $\alpha=0$. Thus $x=y \times z$.

Conversely, let $u_{1}, \cdots, u_{n}$ be an orthogonal basis of $V$. Fix $i$ and let $z^{L}=u_{i} \times z$ for $z \in V$. The assumption implies $u_{k}$ is in the image of $L$ for all $k \neq i . \quad V$ is division-like, so $u_{i} \neq u_{i} \times z$ for any $z \in V$, which implies the set of vectors $\left\{u_{k} \mid k \neq i\right\}$ spans the image of $L$. Hence $u_{j}^{L}=u_{i} \times u_{j}=\sum_{k \neq i} \xi_{i j k} u_{k}$, which implies $\xi_{i j i}=0$ for all $j=1, \cdots, n$. The arbitrariness of $i$ gives the desired conclusion.

We note that if $A \in \mathscr{A}$ is weakly flexible and $x, y \in V$ are such that $(x, y) \neq 0$, then $x=y \times z$ for $z \in V$ is impossible. There exists an orthogonal basis $y=u_{1}, u_{2}, \cdots, u_{n}$ of $V$ and $x=\gamma u_{1}+w$ for $w$ in the span of $\left\{u_{2}, \cdots, u_{n}\right\}$ and $\gamma \neq 0$. Since $A$ is weakly flexible, for any $z \in V$, 
$y \times z$ is in the span of $\left\{u_{2}, \cdots, u_{n}\right\}$. Thus $x=\gamma u_{1}+w=y \times z$ is not possible.

THEOREM 2.1. Let $A \in \mathscr{D}(\mathscr{A})$, $T$ nonsingular on $U$, and $B(A, T)$ flexible. Then $B(A, T) \in \mathscr{D}(\mathscr{A})$.

Proof. Since $(x, y)_{T}=(x, y), B(A, T)$ will be a division algebra, if $V$ is division-like with respect to " $x_{T}$ ". Suppose there exist linearly independent $x$ and $y$ in $V$ such that $x \times_{T} y=x$. The flexibility of $B(A, T)$ implies $\left(x, x \times_{T} y\right)_{T}=0$. Now

$$
x^{2}=x \cdot x=x \cdot\left(x \times_{T} y\right)=\left(x, x \times_{T} y\right)_{T}+x \times_{T}\left(x \times_{T} y\right)=0+x \times_{T} x=0,
$$

which contradicts the assumption that $A \in \mathscr{D}(\mathscr{A})$. Suppose there exist linearly independent $x$ and $y$ in $V$ such that $x \times_{T} y=0$. Then by (1.4), $0=x \times_{T} y=(x \times y)^{T}$. But $T$ is nonsingular, so $x \times y=0$ which also contradicts $A \in \mathscr{D}(\mathscr{A})$.

If $1, u_{1}, u_{2}, \cdots, u_{n}$ is an orthogonal basis of $A \in \mathscr{D}(\mathscr{A})$, then $u_{1} \times$ $x \neq 0$ for $x$ in the span of $\left\{u_{2}, \cdots, u_{n}\right\}$. Thus the $n-1$ vectors $u_{1} \times u_{2}$, $u_{1} \times u_{3}, \cdots, u_{1} \times u_{n}$ are linearly independent. Moreover, since $V$ is division-like, we cannot have

$$
u_{1}=\sum_{i=2}^{n} \beta_{i}\left(u_{1} \times u_{i}\right)=u_{1} \times \sum_{i=2}^{n} \beta_{i} u_{i}
$$

so the $n$ vectors $u_{1}, u_{1} \times u_{2}, u_{1} \times u_{3}, \cdots, u_{1} \times u_{n}$ are linearly independent. Let $v$ be any vector such that $\left(u_{1}, v\right)=0$. If $A$ were weakly flexible, then by Lemma 2.2 there exists $z \in V$ such that $u_{1}=v \times z$ which puts $u_{1} \in U$. Thus $U$ is a $n$-dimensional space contained in $V$, if $n>1$. Hence it is plausible to assume $T$ is a linear transformation from $V$ into $V$.

Corollary 2.1. Let $A$ be flexible and in $\mathscr{A}$ but not in $\mathscr{D}(\mathscr{A})$. Then $B(A, T)$ is not in $\mathscr{D}(\mathscr{A})$ for any nonsingular $T: V \rightarrow V$.

Proof. Since $T$ is nonsingular on $V, T^{-1}: V \rightarrow V$ exists and it is easily checked that $A=B\left(B(A, T), T^{-1}\right)$. So by Theorem 2.1, if $B(A, T)$ were in $\mathscr{D}(\mathscr{A})$, then $A$ would have to also be in $\mathscr{D}(\mathscr{A})$.

THEOREM 2.2. Let $A \in \mathscr{A}$ and suppose that for all $x \in U$, there exist $y$ and $z \in V$ such that $x=y \times z$. If $T$ is singular on $U$, then $B(A, T)$ is not a division algebra.

Proof. $T$ singular implies there exists $x \neq 0$ in $U$ such that $x^{T}=0$. Choose $y$ and $z \in V$ such that $x=y \times z$. Then $0=x^{T}=$ 
$(y \times z)^{T}=y \times{ }_{T} z$, which implies $B(A, T)$ is not a division algebra since $V$ is not division-like with respect to " $X_{T}$ ".

By Lemma 2.2 the condition on $V$ in Theorem 2.2 holds in particular if $A \in \mathscr{D}(\mathscr{A})$ is weakly flexible and $n>1$. If $n=1$, then $U=0$. Thus for $A \in \mathscr{D}(\mathscr{A})$ weakly flexible and $B(A, T)$ flexible we have, by Theorems 2.1 and $2.2, B(A, T) \in \mathscr{D}(\mathscr{A})$ if and only if $T$ is nonsingular on $V$.

If we assume $A \in \mathscr{D}(\mathscr{A})$ is flexible and that $T$ is a scalar $\delta$ times the identity transformation $I$ on $V$, then for any orthogonal basis $u_{1}, \cdots, u_{n}$ of $V$ we have

$$
\sum_{k=1}^{n} \beta_{i j k} u_{k}=u_{i} \times_{T} u_{j}=\left(u_{i} \times u_{j}\right)^{T}=\sum_{k=1}^{n} \xi_{i j k} u_{k}^{T}=\sum_{k=1}^{n} \xi_{i j k} \delta u_{k} .
$$

So $\beta_{i j k}=\xi_{i j k} \delta$ for all $i, j, k=1, \cdots, n$. Since $A$ is flexible, the $\beta_{i j k}$ clearly satisfy the conditions in Lemma 2.1 which make $B(A, T)$ flexible and then by Theorem $2.1 B(A, T) \in \mathscr{D}(\mathscr{A})$.

THEOREM 2.3. Let $A$ be weakly flexible in $\mathscr{D}(\mathscr{A})$ and let $T: V \rightarrow V$ be such that $B(A, T)$ is weakly flexible. Then $T$ is a scalar multiple of $I$.

Proof. Let $u_{1}, \cdots, u_{n}$ be an orthogonal basis of $V$. Pick $u_{r}$ and $u_{s}$ such that $r \neq s . \quad A$ is weakly flexible, so by Lemma 2.2 there exists $z \in V$ such that $u_{s}=u_{r} \times z$. Suppose $u_{s}^{T}=\sum_{l=1 s}^{n} \delta_{l} u_{l}$ and $z=\sum_{j=1}^{n} \gamma_{j} u_{j}$. Then

$$
\sum_{l=1}^{n}{ }_{s} \delta_{l} u_{l}=\left(u_{r} \times z\right)^{T}=\sum_{j=1}^{n} \gamma_{j}\left(u_{r} \times u_{j}\right)^{T}=\sum_{l=1}^{n} \sum_{j=1}^{n} \gamma_{j} \beta_{r j l} u_{l} .
$$

So ${ }_{s} \delta_{r}=\sum_{j=1}^{n} \gamma_{j} \beta_{r j r}=0$, since $\beta_{r i r}=0$ for all $r$ and $j$. Thus $u_{s}^{T}={ }_{s} \delta_{s} u_{s}=\delta_{s} u_{s}$ for each $s=1, \cdots, n$. The extra subscript is now dropped for the sake of simplicity. To show $T$ is a scalar multiple of the identity let $u_{1}$ be any nonzero element of $V$. Then $u_{1}$ may be embedded in a basis $u_{1}, \cdots, u_{n}$ of $V$ and we have $u_{1}^{T}=\delta_{1} u_{1}$ for some $\delta_{1} \in F$. Then also for any $v \in V$, $v^{T}=\delta_{2} v$ for some $\delta_{2} \in F$, and $\delta_{1} u_{1}+\delta_{2} v=u_{1}^{T}+v^{T}=\left(u_{1}+v\right)^{T}=$ $\delta_{3}\left(u_{1}+v\right)=\delta_{3} u_{1}+\delta_{3} v$ for some $\delta_{3} \in F$. Hence $\delta_{1}=\delta_{2}=\delta_{3}$ and $T=\delta_{1} I$.

Since the Cayley-Dickson algebras are alternative, they are flexible. So for $A$ a Cayley-Dickson algebra in $\mathscr{D}(\mathscr{A}), B(A, T)$ is flexible if and only if $T$ is a scalar times $I$, the identity transformation on $V$.

Corollary 2.2. Let $A$ be a Cayley-Dickson algebra in $\mathscr{D}(\mathscr{A})$. $B(A, T)$ is a Cayley-Dickson algebra in $\mathscr{D}(\mathscr{A})$ if and only if $T= \pm I$. 
Proof. It is easily checked that a quadratic algebra is alternative if and only if it is flexible and $(y, x) x-(x, x) y+(y \times x) \times x=0$ for all vectors $x$ and $y$ in the algebra. All that remains to be shown is that in $B(A, T),(y, x)_{T} x-(x, x)_{T} y+\left(y \times_{T} x\right) \times_{T} x=0$ if and only if $T= \pm I . \quad$ By (1.4) and Theorem 2.3

$$
\begin{aligned}
(y, x)_{T} x-(x, x)_{T} y+\left(y \times_{T} x\right) \times_{T} x & =(y, x) x-(x, x) y+\left[(y \times x)^{T} \times x\right]^{T} \\
& =(y, x) x-(x, x) y+\delta^{2}(y \times x) \times x
\end{aligned}
$$

for some scalar $\delta$. Since $A$ is alternative, this expression is 0 if and only if $\delta^{2}=1$.

3. In this section the bonding mapping process is applied to a class of Cayley-Dickson division algebras over formally real fields to obtain nonflexible quadratic division algebras of dimension 8 . We use the definition, as given by Kleinfeld [4], of a Cayley-Dickson algebra in terms of its multiplication table with respect to a basis $1, u_{1}, \cdots, u_{7}$ and parameters $\alpha, \beta$, and $\gamma$. Exact conditions on $\alpha, \beta, \gamma$, and the field $F$ which make the algebra a division algebra are given by Schafer [7]. We consider only the Cayley-Dickson division algebras over formally real fields with $\alpha=\beta=\gamma=-1$. (The Cayley numbers are in this class.) The multiplication table for the nonidentity basis elements in such an algebra $A=F+V$ is given in Table I. It is clear by Table $I$ that for such a

TABLE I

\begin{tabular}{llllllll}
\hline & $u_{1}$ & $u_{2}$ & $u_{3}$ & $u_{4}$ & $u_{5}$ & $u_{6}$ & $u_{7}$ \\
\hline$u_{1}$ & -1 & $u_{3}$ & $-u_{2}$ & $u_{5}$ & $-u_{4}$ & $-u_{7}$ & $u_{6}$ \\
\hline$u_{2}$ & $-u_{3}$ & -1 & $u_{1}$ & $u_{6}$ & $u_{7}$ & $-u_{4}$ & $-u_{5}$ \\
\hline$u_{3}$ & $u_{2}$ & $-u_{1}$ & -1 & $u_{7}$ & $-u_{6}$ & $u_{5}$ & $-u_{4}$ \\
\hline$u_{4}$ & $-u_{5}$ & $-u_{6}$ & $-u_{7}$ & -1 & $u_{1}$ & $u_{2}$ & $u_{3}$ \\
\hline$u_{5}$ & $u_{4}$ & $-u_{7}$ & $u_{6}$ & $-u_{1}$ & -1 & $-u_{3}$ & $u_{2}$ \\
\hline$u_{6}$ & $u_{7}$ & $u_{4}$ & $-u_{5}$ & $-u_{2}$ & $u_{3}$ & -1 & $-u_{1}$ \\
\hline$u_{7}$ & $-u_{6}$ & $u_{5}$ & $u_{4}$ & $-u_{3}$ & $-u_{2}$ & $u_{1}$ & -1 \\
\hline
\end{tabular}

Cayley-Dickson algebra $u_{1}, \cdots, u_{7}$ is an orthogonal basis for $V$, and that each $u_{i}$ for $i=1, \cdots, 7$ is equal to $u_{j} \times u_{k}$ for some $j, k \in\{1, \cdots, 7\}$, so that the subspace $U$ as defined in $\S 1$ is equal to $V$. Moreover, $\alpha_{i}=\left(u_{i}, u_{i}\right)=$ -1 for $i=1, \cdots, 7$. A is the special case $\tau=0$ of the class of division 
algebras we are about to define. Let $T$ be a bonding mapping from $V$ to $V$ with matrix representation

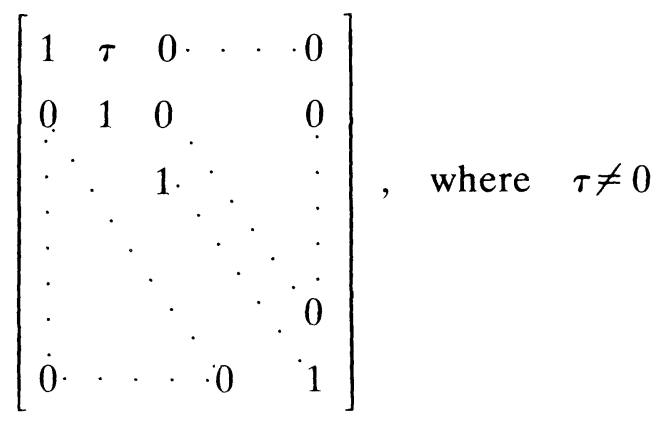

is in $F$, with respect to the basis $u_{1}, \cdots, u_{7}$. By (1.4) the multiplication in $B(A, T)$ of two nonidentity basis elements is given by $u_{i} \cdot u_{j}=$ $\left(u_{i}, u_{j}\right)+\left(u_{i} \times y_{j}\right)^{T}$, where $(x, y)$ is the bilinear form determined by $A$ and " $x$ " is the skew-commutative multiplication in $V$ determined by $A$. So

$$
\begin{aligned}
u_{i} \cdot u_{i} & =\left(u_{i}, u_{i}\right)+\left(u_{i} \times u_{i}\right)^{T} \\
& =\left(u_{i}, u_{i}\right)=-1 \text { for } i=1, \cdots, 7 \\
u_{i} \cdot u_{j} & =\left(u_{i}, u_{j}\right)+\left(u_{i} \times u_{j}\right)^{T} \\
& =\left(u_{i} \times u_{i}\right)^{T} \quad \text { for } \quad i \neq j ; \quad i, j=1, \cdots, 7 .
\end{aligned}
$$

Using (3.2) one obtains the multiplication table for $B(A, T)$ given in Table II.

TABLE II

\begin{tabular}{cccccccc}
\hline & $u_{1}$ & $u_{2}$ & $u_{3}$ & $u_{4}$ & $u_{5}$ & $u_{6}$ & $u_{7}$ \\
\hline$u_{1}$ & -1 & $u_{3}$ & $-u_{2}$ & $u_{5}$ & $-u_{4}$ & $-u_{7}$ & $u_{6}$ \\
\hline$u_{2}$ & $-u_{3}$ & -1 & $u_{1}+\tau u_{2}$ & $u_{6}$ & $u_{7}$ & $-u_{4}$ & $-u_{5}$ \\
\hline$u_{3}$ & $u_{2}$ & $-u_{1}-\tau u_{2}$ & -1 & $u_{7}$ & $-u_{6}$ & $u_{5}$ & $-u_{4}$ \\
\hline$u_{4}$ & $-u_{5}$ & $-u_{6}$ & $-u_{7}$ & -1 & $u_{1}+\tau u_{2}$ & $u_{2}$ & $u_{3}$ \\
\hline$u_{5}$ & $u_{4}$ & $-u_{7}$ & $u_{6}$ & $-u_{1}-\tau u_{2}$ & -1 & $-u_{3}$ & $u_{2}$ \\
\hline$u_{6}$ & $u_{7}$ & $u_{4}$ & $-u_{5}$ & $-u_{2}$ & $u_{3}$ & -1 & $-u_{1}-\tau u_{2}$ \\
\hline$u_{7}$ & $-u_{6}$ & $u_{5}$ & $u_{4}$ & $-u_{3}$ & $-u_{2}$ & $u_{1}+\tau u_{2}$ & -1 \\
\hline
\end{tabular}

We shall prove that $B(A, T)$ is a division algebra for any $T$ as in (3.1) such that $|\tau|<2$, and we shall give examples of zero divisors when $\tau=2$.

We take $T$ in (3.1) such that $|\tau|<2$ and let 


$$
x=a u_{0}+b u_{1}+c u_{2}+d u_{3}+f u_{4}+g u_{5}+h u_{6}+k u_{7}
$$

and

$$
y=a^{\prime} u_{0}+b^{\prime} u_{1}+c^{\prime} u_{2}+d^{\prime} u_{3}+f^{\prime} u_{4}+g^{\prime} u_{5}+h^{\prime} u_{6}+k^{\prime} u_{7},
$$

where $a, \cdots, k, a^{\prime}, \cdots, k^{\prime} \in F$ be two aribtrary elements of $B(A, T)$. Using Table II we can express the relation $x \cdot y=0$ in terms of the basis elements $1, u_{1}, \cdots, u_{7}$ for $B(A, T)$ in the following way:

$$
\begin{aligned}
0= & \left(a a^{\prime}-b b^{\prime}-c c^{\prime}-d d^{\prime}-f f^{\prime}-g g^{\prime}-h h^{\prime}-k k^{\prime}\right) 1 \\
& +\left(a b^{\prime}+b a^{\prime}+c d^{\prime}-d c^{\prime}+f g^{\prime}-g f^{\prime}+k h^{\prime}-h k^{\prime}\right) u_{1} \\
& +\left(a c^{\prime}+c a^{\prime}+d b^{\prime}-b d^{\prime}+\tau c d^{\prime}-\tau d c^{\prime}+\tau f g^{\prime}\right. \\
& \left.-\tau g f^{\prime}+f h^{\prime}-h f^{\prime}+g k^{\prime}-k g^{\prime}+\tau k h^{\prime}-\tau h k^{\prime}\right) u_{2} \\
& +\left(a d^{\prime}+d a^{\prime}+b c^{\prime}-c b^{\prime}+f k^{\prime}-k f^{\prime}+h g^{\prime}-g h^{\prime}\right) u_{3} \\
& +\left(a f^{\prime}+f a^{\prime}+g b^{\prime}-b g^{\prime}+h c^{\prime}-c h^{\prime}+k d^{\prime}-d k^{\prime}\right) u_{4} \\
& +\left(a g^{\prime}+g a^{\prime}+b f^{\prime}-f b^{\prime}+k c^{\prime}-c k^{\prime}+d h^{\prime}-h d^{\prime}\right) u_{5} \\
& +\left(a h^{\prime}+h a^{\prime}+b k^{\prime}-k b^{\prime}+c f^{\prime}-f c^{\prime}+g d^{\prime}-d g^{\prime}\right) u_{6} \\
& +\left(a k^{\prime}+k a^{\prime}+h b^{\prime}-b h^{\prime}+c g^{\prime}-g c^{\prime}+d f^{\prime}-f d^{\prime}\right) u_{7} .
\end{aligned}
$$

This gives eight homogeneous bilinear equations in the elements $a, \cdots, k, a^{\prime}, \cdots, k^{\prime}$. The equation $x \cdot y=0$ has a solution in $B(A, T)$ if and only if these eight equations can be made to equal zero simultaneously. We way think of the primed letters $a^{\prime}, \cdots, k^{\prime}$ as variables and consider the coefficient matrix $M_{T}$ of the set of eight equations. We have

$$
M_{T}=\left[\begin{array}{rrrrrrrr}
a & -b & -c & -d & -f & -g & -h & -k \\
b & a & -d & c & -g & f & k & -h \\
c & d & a-\tau d & -b+\tau c & -h-\tau g & -k+\tau f & f+\tau k & g-\tau h \\
d & -c & b & a & -k & h & -g & f \\
f & g & h & k & a & -b & -c & -d \\
g & -f & k & -h & b & a & d & -c \\
h & -k & -f & g & c & -d & a & b \\
k & h & -g & -f & d & c & -b & a
\end{array}\right] .
$$

It suffices to show this matrix is nonsingular for all choices of $a, \cdots, k$ not all zero. To show $M_{T}$ is nonsingular for $|\tau|<2$ we utilize a technique found in [6]. Let 


$$
M_{T}^{\prime}=\left[\begin{array}{rrrrrrrr}
a & b & c & d & f & g & h & k \\
-b & a & d & -c & g & -f & -k & h \\
-c & -d & a & b & h & k & -f & -g \\
-d & c & -b & a & k & -h & g & -f \\
-f & -g & -h & -k & a & b & c & d \\
-g & f & -k & h & -b & a & -d & c \\
-h & k & f & -g & -c & d & a & -b \\
-k & -h & g & f & -d & -c & b & a
\end{array}\right] .
$$

If we set $\Gamma=a^{2}+b^{2}+c^{2}+d^{2}+f^{2}+g^{2}+h^{2}+k^{2}$, then

$M_{\mathrm{T}} M_{T}^{\prime}=$

$\left[\begin{array}{cccccccc}\Gamma & 0 & 0 & 0 & 0 & 0 & 0 & 0 \\ 0 & \Gamma & 0 & 0 & 0 & 0 & 0 & 0 \\ 0 & \tau\left(\Gamma-a^{2}-b^{2}\right) & \Gamma-\tau(a d+b c) & \tau(a c-b d) & -\tau(a g+b f) & \tau(a f-b g) & \tau(a k-b h) & \tau(a h-b h) \\ 0 & 0 & 0 & \Gamma & 0 & 0 & 0 & 0 \\ 0 & 0 & 0 & 0 & \Gamma & 0 & 0 & 0 \\ 0 & 0 & 0 & 0 & 0 & \Gamma & 0 & 0 \\ 0 & 0 & 0 & 0 & 0 & 0 & \Gamma & 0 \\ 0 & 0 & 0 & 0 & 0 & 0 & 0 & \Gamma\end{array}\right]$

Any choice of $\tau$ which makes $M_{T} M_{T}^{\prime}$ nonsingular will clearly make $M_{T}$ nonsingular. We have

$$
\operatorname{det} M_{T} M_{T}^{\prime}=\Gamma^{7}[\Gamma-\tau(a d+b c)]
$$

Since $F$ is a formally real field, $\Gamma>0$ in $F$ unless $a=b=c=d=f=g=$ $h=k=0$. We expand the other factor of $\operatorname{det} M_{T} M_{T}^{\prime}$ to obtain

$$
\begin{aligned}
\Gamma-\tau(a d+b c)= & a^{2}-\tau a d+d^{2}+b^{2}-\tau b c+c^{2} \\
& +f^{2}+g^{2}+h^{2}+k^{2} .
\end{aligned}
$$

We want to show the expression in (3.4) is nonzero for any $\tau \in F$ such that $|\tau|<2$. Consider the quadratic form $q=\lambda_{1}^{2}+\tau \lambda_{1} \lambda_{2}+\lambda_{2}^{2}$ and the nonsingular linear transformation given by $\lambda_{1}=\mu_{1}-\mu_{2}$ and $\lambda_{2}=\mu_{1}+\mu_{2}$. This transformation applied to $q$ gives a new quadratic form $p=$ $(2+\tau) \mu_{1}^{2}+(2-\tau) \mu_{2}^{2}$. Since the transformation connecting them is nonsingular, $q$ and $p$ are congruent. Therefore, they have the same range of 
values when $\lambda_{1}, \lambda_{2}$ and $\mu_{1}, \mu_{2}$ assume all values in the formally real field F. But $|\tau|<2$ implies $2+\tau>0$ and $2-\tau>0$. So $p>0$ which implies $q>0$ for $|\tau|<2$. Applying this conclusion to (3.4) shows $\Gamma-$ $\tau(a d+b c)>0$ for $|\tau|<2$. Thus $M_{T} M_{T}^{\prime}$ and $M_{T}$ are nonsingular and $B(A, T)$ has no nontrivial zero divisors.

Let $T_{0}$ be the nonsingular linear transformation obtained by setting $\tau=2$ in (3.1). $B\left(A, T_{0}\right)$ will have divisors of zero. The multiplication table for $B\left(A, T_{0}\right)$ is Table II with $\tau=2$. Let $M_{T_{0}}$ be the matrix obtained from $M_{T}$ by setting $\tau=2$. It is easily seen that det $M_{T_{0}}=0$ for $a=d$, $b=c$, and $f=g=h=k=0$, so that nontrivial solutions to $x \cdot y=0$ do exist in $B\left(A, T_{0}\right)$. (E.g. $x=1+u_{1}+u_{2}+u_{3}$ and $y=1+u_{1}+u_{2}-u_{3}$ have product 0 in $B\left(A, T_{0}\right)$.)

Albert [1], Bruck [3], and Osborn [6] have constructed classes of quadratic division algebras. A full determination of quadratic division algebras obtainable by this bonding mapping process has not been made even when $A$ is taken to be a Cayley-Dickson algebra. The class of division algebras obtained above with $\tau \neq 0$ does not contain any flexible algebras, since $u_{2} \cdot u_{3}=u_{1}+\tau u_{2}$ with $\tau \neq 0$ violates condition (a) of Lemma 2.1. Moreover, for $T$ as in (3.1) with $\tau \neq 0$ one obtains $u_{1} \times_{T} u_{4}=$ $u_{5}, u_{4} \times_{T} u_{5}=u_{1}+\tau u_{2}, u_{1} \times_{T} u_{2}=u_{3}, u_{2} \times_{T} u_{4}=u_{6}$, and $u_{2} \times_{T} u_{5}=u_{7}$, so that the skew-commutative algebra generated in $V$ by $u_{1}$ and $u_{4}$ is $V$ itself. This shows that no $B(A, T)$ obtained as above with $\tau \neq 0$ is a division algebra of dimension 8 in the class discovered by Osborn [6], since in his class of examples every two independent elements in $V$ generate a subalgebra in $V$ of dimension 3 .

\section{REFERENCES}

1. A. A. Albert, Non-associative algebras, II, New simple algebras, Annals of Math., 43 (1942), 708-723.

2. - New simple power associative algebras, Summa Brasiliensis Mathematicae, 2 (1951), $1-12$.

3. R. H. Bruck, Some results in the theory of linear non-associative algebras, Trans. Amer. Math. Soc., 56 (1944), 141-199.

4. E. Kleinfeld, Studies in Modern Algebra, M.A.A. Studies in Mathematics, Prentice-Hall, New Jersey, 2 (1963); 126-143.

5. R. H. Oehmke, A class of noncommutative power associative algebras, Trans. Amer. Math. Soc., 87 (No. 1), (1958), 226-236.

6. J. M. Osborn, Quadratic division algebras, Trans. Amer. Math. Soc., 105 (1962), 202-221.

7. R. D. Schafer, Alternative algebras over an arbitrary field, Bull. Amer. Math. Soc., 49 (1943), $549-555$.

Received November 5, 1975. A portion of the results herein were submitted to the Graduate School of the University of Iowa as part of the author's dissertation supervised by Professor R. H. Oehmke. 



\section{PACIFIC JOURNAL OF MATHEMATICS}

\section{EDITORS}

RICHARD ARENS (Managing Editor)

University of California

Los Angeles, California 90024

\author{
R. A. Beaumont \\ University of Washington \\ Seattle, Washington 98105
}

\section{J. DugunduI}

Department of Mathematics University of Southern California Los Angeles, California 90007

D. Gilbarg and J. Milgram

Stanford University

Stanford, California 94305

\section{ASSOCIATE EDITORS}

E. F. BECKENBACH
B. H. NeumanN

F. Wolp
K. YoshidA

\section{SUPPORTING INSTITUTIONS}

UNIVERSITY OF BRITISH COLUMBIA

CALIFORNIA INSTITUTE OF TECHNOLOGY

UNIVERSITY OF CALIFORNIA

MONTANA STATE UNIVERSITY

UNIVERSITY OF NEVADA

NEW MEXICO STATE UNIVERSITY

OREGON STATE UNIVERSITY

UNIVERSITY OF OREGON

OSAKA UNIVERSITY

\author{
UNIVERSITY OF SOUTHERN CALIFORNIA \\ STANFORD UNIVERSITY \\ UNIVERSITY OF HAWAII \\ UNIVERSITY OF TOKYO \\ UNIVERSITY OF UTAH \\ WASHINGTON STATE UNIVERSITY \\ UNIVERSITY OF WASHINGTON \\ AMERICAN MATHEMATICAL SOCIETY
}

The Supporting Institutions listed above contribute to the cost of publication of this Journal, but they are not owners or publishers and have no responsibility for its contents or policies.

Mathematical papers intended for publication in the Pacific Journal of Mathematics should be in typed form or offset-reproduced (not dittoed). double spaced with large margins. Underline Greek letters in red, German in green, and script in blue. The first paragraph or two must be capable of being used separately as a synopsis of the entire paper. Items of the biblography should not be cited there unless absolutely necessary, in which case they must be identified by author and Journal, rather than by item number. Manuscripts, in duplicate, may be sent to any one of the four editors. Please classify according to the scheme of Math. Reviews, Index to Vol. 39. All other communications should be addressed to the managing editor, or Elaine Barth, University of California, Los Angeles, California, 90024.

100 reprints are provided free for each article, only if page charges have been substantially paid. Additional copies may be obtained at cost in multiples of 50 .

The Pacific Journal of Mathematics is issued monthly as of January 1966. Regular subscription rate: $\$ 72.00$ a year (6 Vols., 12 issues). Special rate: $\$ 36.00$ a year to individual members of supporting institutions.

Subscriptions, orders for back numbers, and changes of address should be sent to Pacific Journal of Mathematics, 103 Highland Boulevard, Berkeley, California, 94708.

PUBLISHED BY PACIFIC JOURNAL OF MATHEMATICS, A NON-PROFIT CORPORATION Printed at Jerusalem Academic Press, POB 2390, Jerusalem, Israel.

Copyright (C) 1976 Pacific Journal of Mathematics All Rights Reserved 


\section{Pacific Journal of Mathematics}

\section{Vol. 64, No. $2 \quad$ June, 1976}

Richard Fairbanks Arnold and A. P. Morse, Plus and times............. 297

Edwin Ogilvie Buchman and F. A. Valentine, External visibility ......... 333

R. A. Czerwinski, Bonded quadratic division algebras.............. 341

William Richard Emerson, Averaging strongly subadditive set functions in unimodular amenable groups. II .........................

Lynn Harry Erbe, Existence of oscillatory solutions and asymptotic behavior for a class of third order linear differential equations ............. 369

Kenneth R. Goodearl, Power-cancellation of groups and modules........ 387

J. C. Hankins and Roy Martin Rakestraw, The extremal structure of locally compact convex sets ...................................

Burrell Washington Helton, The solution of a Stieltjes-Volterra integral

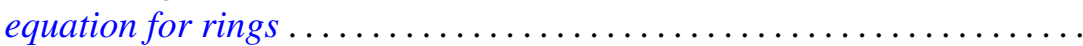

Frank Kwang-Ming Hwang and Shen Lin, Construction of 2-balanced

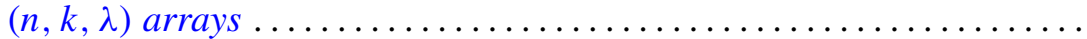

Wei-Eihn Kuan, Some results on normality of a graded ring ... 455

Dieter Landers and Lothar Rogge, Relations between convergence of series and convergence of sequences ......................... 465

Lawrence Louis Larmore and Robert David Rigdon, Enumerating immersions and embeddings of projective spaces ................

Douglas C. McMahon, On the role of an abelian phase group in relativized problems in topological dynamics..................

Robert Wilmer Miller, Finitely generated projective modules and TTF classes...

Yashaswini Deval Mittal, A class of isotropic covariance functions ...

Anthony G. Mucci, Another martingale convergence theorem ...

Joan Kathryn Plastiras, Quasitriangular operator algebras ...

John Robert Quine, Jr., The geometry of $p\left(S^{1}\right) \ldots \ldots \ldots$. 